\title{
Convergence Analysis of Iterative Scheme and Error Estimation of Positive Solution for a Fractional Differential Equation
}

\section{Jing $\mathbf{W u}^{a}$, Xinguang Zhang ${ }^{b, d}$, Lishan $\mathbf{L i u}^{c, d}$, Yonghong $\mathbf{W} \mathbf{u}^{d}$ and Yujun $\mathrm{Cui}^{e}$}

${ }^{a}$ School of Economic Mathematics, Southwestern University of Finance and Economics

Chengdu, 610074 Sichuan, China

${ }^{b}$ School of Mathematical and Informational Sciences, Yantai University

Yantai, 264005 Shandong, China

${ }^{c}$ School of Mathematical Sciences, Qufu Normal University

Qufu, 273165 Shandong, China

${ }^{d}$ Department of Mathematics and Statistics, Curtin University of Technology Perth, 6845 WA, Australia

${ }^{e}$ Department of Mathematics, Shandong University of Science and Technology Qingdao, 266590 Shandong, China

E-mail(corresp.): zxg123242@163.com

Received October 23, 2017; revised September 7, 2018; accepted September 10, 2018

\begin{abstract}
In this paper, we focus on the iterative scheme and error estimation of positive solutions for a class of $p$-Laplacian fractional order differential equation subject to Riemann-Stieltjes integral boundary condition. Under a weaker growth condition of nonlinearity, by using a monotone iterative technique, we first establish a new result on the sufficient condition for the existence of a unique positive solution to the above problem, then construct an iterative scheme which converges to the unique positive solution, and then present an error estimation and the exact convergence rate of the approximate solution.
\end{abstract}

Keywords: uniqueness, fractional $p$-Laplacian equation, monotone iterative technique, error estimation.

AMS Subject Classification: 34B15; 34B25.

Copyright (c) 2018 The Author(s). Published by VGTU Press

This is an Open Access article distributed under the terms of the Creative Commons Attribution License (http://creativecommons.org/licenses/by/4.0/), which permits unrestricted use, distribution, and reproduction in any medium, provided the original author and source are credited. 


\section{Introduction}

Fractional order differential equations are generalization of integer order differential equations to non-integer order ones. Due to the nonlocality of fractional order derivatives, the generalization has a wide range of interesting applications in science and engineering. For example, many physical phenomena with long time memory behavior in diffusive interaction, electrochemistry, polymer rheology can be described successfully by fractional order mathematical models $[1,18,26,27,31,36,41]$. Thus fractional differential equations are regarded as an excellent tool for modeling various complex processes in natural sciences and engineering such as bioprocess [15,37] and viscoelasticity $[11,22,25,40,42]$.

In this paper, we focus on the iterative scheme and error estimation of positive solutions for the following $p$-Laplacian fractional order differential equation subject to Riemann-Stieltjes integral boundary condition

$$
\left\{\begin{array}{l}
-\mathcal{D}_{\boldsymbol{t}}^{\beta}\left(\varphi_{p}\left(-\mathcal{D}_{\boldsymbol{t}}{ }^{\alpha} z\right)\right)(t)=f\left(t, z(t), \mathcal{D}_{\boldsymbol{t}}^{\gamma} z(t)\right), \quad t \in(0,1), \\
\mathcal{D}_{\boldsymbol{t}}^{\alpha} z(0)=\mathcal{D}_{\boldsymbol{t}}{ }^{\alpha+1} z(0)=\mathcal{D}_{\boldsymbol{t}}^{\gamma} z(0)=0, \\
\mathcal{D}_{\boldsymbol{t}}^{\alpha} z(1)=0, \mathcal{D}_{\boldsymbol{t}}^{\gamma} z(1)=\int_{0}^{1} \mathcal{D}_{\boldsymbol{t}}^{\gamma} z(s) d A(s),
\end{array}\right.
$$

where $\mathcal{D}_{\boldsymbol{t}}{ }^{\alpha}, \mathcal{D}_{\boldsymbol{t}}{ }^{\beta}, \mathcal{D}_{\boldsymbol{t}}^{\gamma}$ are the Riemann-Liouville fractional derivatives of order $\alpha, \beta, \gamma$ with $0<\gamma \leq 1<\alpha \leq 2<\beta<3, \alpha-\gamma>1, \int_{0}^{1} \mathcal{D}_{\boldsymbol{t}}^{\gamma} z(s) d A(s)$ denotes a Riemann-Stieltjes integral, $A$ is a function of bounded variation. The $p$ Laplacian operator is defined as $\varphi_{p}(s)=|s|^{p-2} s, p>2$, and obviously, $\varphi_{p}(s)$ is invertible and its inverse operator is $\varphi_{q}(s)$, where $q=\frac{p}{p-1}$ is the conjugate index of $p$.

In recent years, many nonlinear functional analysis methods have been developed to solve local or nonlocal nonlinear boundary value problems of differential equations, such as the iterative methods $[2,8,10,12,17,19,20,23,24,28$, $29,30,32,34,38]$, upper and lower solution methods [17, 22, 23], the variational method $[7,8,9,13,33]$ and the fixed point theorems $[4,5,6,14,24,35,39]$. These tools and methods not only serve for various applied mathematics problems but also are used to solve many practical problems from physics and engineering. In [16], Webb and Zima established the existence of multiple positive solutions for the nonlocal boundary value problem

$$
\left\{\begin{array}{l}
z^{\prime \prime}(t)+k^{2} z=f(t, z(t)), \quad 0<t<1, \\
z(0)=0, z(1)=\int_{0}^{1} z(s) d A(s),
\end{array}\right.
$$

where $d A$ can be a signed measure. By means of the monotone iterative technique, Mao et. al [10] considered the uniqueness of the positive solution for the nonlocal problem (1.2) under the following growth condition:

$\left(\mathbf{H}_{1}\right) f(t, z)$ is nondecreasing in $z$, and there exists a constant $0<b<1$ such that, for any $(t, x) \in(0,1) \times[0,+\infty)$ and $r \in(0,1)$,

$$
f(t, r z) \geq r^{b} f(t, z) .
$$


In [21], a more general condition is used to obtain a necessary and sufficient condition of positive solutions for a nonlinear singular differential system, namely

$\left(\mathbf{H}_{2}\right) f(t, z)$ is nondecreasing in $z$; for any $r \in(0,1)$, there exists $\eta(r)=$ $m\left(r^{-\kappa}-1\right)$ such that, for all $(t, z) \in(0,1) \times[0,+\infty), 0<m \leq 1,0<\kappa<1$,

$$
f(t, r z) \geq r(1+\eta(r)) f(t, z) .
$$

The aim of this paper is to find weaker conditions than $\left(\mathbf{H}_{1}\right)$ and $\left(\mathbf{H}_{2}\right)$, and then to establish the uniqueness of the positive solution for the general $p$-Laplacian fractional order differential equation (1.1). The iterative scheme converging to the unique solution will also be constructed. Based on the convergence sequence, estimates on the error and the convergence rate of approximate solution are also obtained.

\section{Preliminaries and lemmas}

Before we give a detailed description of some preliminaries and lemmas, we first introduce a weaker condition than $\left(\mathbf{H}_{1}\right)$ and $\left(\mathbf{H}_{2}\right)$, which is to be used in the rest of the paper.

(A) $f:((0,1) \times[0,+\infty) \times[0,+\infty) \rightarrow[0,+\infty))$ is continuous and nondecreasing in the second and the third variables; and for any $(t, x, y) \in(0,1) \times[0,+\infty) \times$ $[0,+\infty)$ and $r \in(0,1)$, there exists a function $\sigma:[0,1] \rightarrow[0,+\infty)$ with $\sigma(r)>r$ for $r \in(0,1)$ such that

$$
f(t, r x, r y) \geq \sigma(r) f(t, x, y) .
$$

Remark 1. Obviously, the assumption (A) includes assumptions $\left(\mathbf{H}_{1}\right)$ and $\left(\mathbf{H}_{2}\right)$ as special cases, that is, the condition (2.1) is weaker than the conditions (1.3) and (1.4).

Remark 2. If $(\mathbf{A})$ holds, then for any $c \geq 1$ and $(t, x, y) \in(0,1) \times[0,+\infty) \times$ $[0,+\infty)$, inequality $(2.1)$ is equivalent to

$$
f(t, c x, c y) \leq \frac{1}{\sigma\left(c^{-1}\right)} f(t, x, y) .
$$

Proof. In fact, for any $c \geq 1$ and $(t, x, y) \in(0,1) \times[0,+\infty) \times[0,+\infty)$, it follows from (2.1) that

$$
f(t, x, y)=f\left(t, c^{-1} c x, c^{-1} c y\right) \geq \sigma\left(c^{-1}\right) f(t, c x, c y),
$$

i.e, inequality (2.2) holds.

Remark 3. The function $f$ includes a large number of nonlinear functions and particularly covers the standard type of sublinear functions

$$
f(t, x, y)=\sum_{i=1}^{n}\left[a_{i}(t) x^{\alpha_{i}}+b_{i}(t) y^{\beta_{i}}\right],
$$

where $0<\alpha_{i}, \beta_{i}<1$ and $a_{i}, b_{i} \in C((0,1),[0,+\infty)), i=1,2, \ldots, n$. 
Now we give some basic notations and concepts on the sense of RiemannLiouville fractional derivatives [11].

The Riemann-Liouville fractional integral of order $\alpha>0$ of a function $x:(0,+\infty) \rightarrow \mathbb{R}$ is given by

$$
I^{\alpha} x(t)=\frac{1}{\Gamma(\alpha)} \int_{0}^{t}(t-s)^{\alpha-1} x(s) d s
$$

provided that the right-hand side is pointwise defined on $(0,+\infty)$.

The Riemann-Liouville fractional derivative of order $\alpha>0$ of a continuous function $x:(0,+\infty) \rightarrow \mathbb{R}$ is given by

$$
\mathcal{D}_{t}^{\alpha} x(t)=\frac{1}{\Gamma(n-\alpha)}\left(\frac{d}{d t}\right)^{(n)} \int_{0}^{t}(t-s)^{n-\alpha-1} x(s) d s,
$$

where $n=[\alpha]+1,[\alpha]$ denotes the integer part of the number $\alpha$, provided that the right-hand side is pointwise defined on $(0,+\infty)$.

In order to simplify the $p$-Laplacian fractional order differential equation (1.1) to a more convenient form, for $x \in C[0,1]$, we make a change of variable by $z(t)=I^{\gamma} x(t)$. Since $1<\alpha \leq 2,1<\alpha-\gamma<2$, according to the definition of the Riemann-Liouville calculus, one has

$$
\begin{gathered}
\mathcal{D}_{\boldsymbol{t}}{ }^{\alpha} z(t)=\frac{d^{2}}{d t^{2}} I^{2-\alpha} z(t)=\frac{d^{2}}{d t^{2}} I^{2-\alpha} I^{\gamma} x(t)=\frac{d^{2}}{d t^{2}} I^{2-\alpha+\gamma} x(t)=\mathcal{D}_{\boldsymbol{t}}{ }^{\alpha-\gamma} x(t), \\
\mathcal{D}_{\boldsymbol{t}}{ }^{\alpha+1} z(t)=\frac{d^{3}}{d t^{3}} I^{3-\alpha-1} z(t)=\frac{d^{3}}{d t^{3}} I^{3-\alpha-1} I^{\gamma} x(t)=\frac{d^{3}}{d t^{3}} I^{3-\alpha-1+\gamma} x(t) \\
=\mathcal{D}_{\boldsymbol{t}}{ }^{\alpha-\gamma+1} x(t), \quad \mathcal{D}_{\boldsymbol{t}}^{\gamma} z(t)=\mathcal{D}_{\boldsymbol{t}}^{\gamma} I^{\gamma} x(t)=x(t) .
\end{gathered}
$$

Consequently, it follows from (2.3) that the $p$-Laplacian fractional order differential equation (1.1) reduces to the following equivalent boundary value problem

$$
\left\{\begin{array}{l}
-\mathcal{D}_{t}^{\beta} \varphi_{p}\left(-\mathcal{D}_{t}^{\alpha-\gamma} x(t)\right)=f\left(t, I^{\gamma} x(t), x(t)\right) \\
\mathcal{D}_{t}^{\alpha-\gamma} x(0)=\mathcal{D}_{t}^{\alpha-\gamma+1} x(0)=\mathcal{D}_{t}^{\alpha-\gamma} x(1)=0 \\
x(0)=0, x(1)=\int_{0}^{1} x(s) d A(s)
\end{array}\right.
$$

Thus in order to establish the existence and uniqueness of positive solutions for the equation (1.1), we only need to focus on the equivalent problem (2.4) of the equation (1.1). To do this, we define

$$
G_{\beta}(t, s)=\frac{1}{\Gamma(\beta)}\left\{\begin{array}{lc}
{[t(1-s)]^{\beta-1},} & 0 \leq t \leq s \leq 1 \\
{[t(1-s)]^{\beta-1}-(t-s)^{\beta-1},} & 0 \leq s \leq t \leq 1
\end{array}\right.
$$

then we have the following lemma:

Lemma 1. (see [22]). Given $g \in L^{1}(0,1)$, the following fractional boundary value problem

$$
\left\{\begin{array}{l}
-\mathcal{D}_{\boldsymbol{t}}{ }^{\alpha-\gamma} x(t)=g(t), \quad 0<t<1, \\
x(0)=x(1)=0
\end{array}\right.
$$


has a unique solution

$$
x(t)=\int_{0}^{1} G_{\alpha-\gamma}(t, s) g(s) d s .
$$

On the other hand, it is easy to know that the fractional differential equation

$$
\left\{\begin{array}{l}
-\mathcal{D}_{t}{ }^{\alpha-\gamma} x(t)=0, \quad 0<t<1, \\
x(0)=0, x(1)=1,
\end{array}\right.
$$

has a unique solution $x(t)=t^{\alpha-\gamma-1}$. Thus according to the strategy of [16], by denoting

$$
\mathcal{A}=\int_{0}^{1} t^{\alpha-\gamma-1} d A(t), \quad \mathcal{G}_{A}(s)=\int_{0}^{1} G_{\alpha-\gamma}(t, s) d A(t),
$$

we can obtain the Green function

$$
H(t, s)=\frac{\mathcal{G}_{A}(s)}{1-\mathcal{A}} t^{\alpha-\gamma-1}+G_{\alpha-\gamma}(t, s)
$$

of the following fractional differential equation subject to the Riemann-Stieltjes integral boundary condition

$$
\left\{\begin{array}{l}
-\mathcal{D}^{\alpha-\gamma} x(t)=g(t) \\
x(0)=0, x(1)=\int_{0}^{1} x(s) d A(s) .
\end{array}\right.
$$

With the help of (2.5)-(2.9), we have the following lemmas which have already been proven in [26].

Lemma 2. Given $g \in L^{1}[0,1], 0<\gamma \leq 1<\alpha \leq 2<\beta<3$ and $\alpha-\gamma>1$, the fractional order $p$-Laplacian differential equation

$$
\left\{\begin{array}{l}
-\mathcal{D}_{\boldsymbol{t}}^{\beta} \varphi_{p}\left(-\mathcal{D}_{\boldsymbol{t}}{ }^{\alpha-\gamma} x(t)\right)=g(t) \\
\mathcal{D}_{\boldsymbol{t}}^{\alpha-\gamma} x(0)=\mathcal{D}_{\boldsymbol{t}}^{\alpha-\gamma+1} x(0)=\mathcal{D}_{\boldsymbol{t}}^{\alpha-\gamma} x(1)=0 \\
x(0)=0, x(1)=\int_{0}^{1} x(s) d A(s)
\end{array}\right.
$$

has a unique solution

$$
x(t)=\int_{0}^{1} H(t, s) \varphi_{q}\left(\int_{0}^{1} G_{\beta}(s, \tau) g(\tau) d \tau\right) d s .
$$

Lemma 3. Assume $0 \leq \mathcal{A}<1$ and $\mathcal{G}_{A}(s) \geq 0$ for $s \in[0,1]$, then the function $G_{\beta}(t, s)$ and $H(t, s)$ enjoy the following properties:

(1) $G_{\beta}(t, s)>0, H(t, s)>0$, for $t, s \in(0,1)$.

(2)

$$
\frac{t^{\beta-1}(1-t) s(1-s)^{\beta-1}}{\Gamma(\beta)} \leq G_{\beta}(t, s) \leq \frac{\beta-1}{\Gamma(\beta)} t^{\beta-1}(1-t), \quad \text { for } t, s \in[0,1] .
$$

(3) There exist two positive constants d, e such that

$$
d t^{\alpha-\gamma-1} \mathcal{G}_{A}(s) \leq H(t, s) \leq e t^{\alpha-\gamma-1}, t, s \in[0,1] .
$$




\section{Main results}

To ensure the validity of Lemma 3, we need the following assumptions.

(B) $A$ is a function of bounded variation satisfying $\mathcal{G}_{A}(s) \geq 0$ for $s \in[0,1]$ and

$$
0 \leq \int_{0}^{1} t^{\alpha-\gamma-1} d A(t)<1
$$

Now we carry out our work in the space $E=C[0,1]$, which is a Banach space endowed with the form $\|x\|=\max _{t \in[0,1]}|x(t)|$ for any $x \in E$. Define

$$
P=\{x \in C[0,1]: x(t) \geq 0, t \in[0,1]\} .
$$

Then $P$ is a normal cone with normality constant 1 in the Banach space $E$. Next define a sub-set of $P$ by

$$
\begin{gathered}
D=\left\{x(t) \in P: \text { there exist two numbers } K_{x}>1>k_{x}>0\right. \text { such that } \\
\left.\qquad k_{x} t^{\alpha-\gamma-1} \leq x(t) \leq K_{x} t^{\alpha-\gamma-1}, t \in[0,1]\right\} .
\end{gathered}
$$

Theorem 1. Suppose the conditions $(\mathbf{A})-(\mathbf{B})$ hold. If

$$
0<\int_{0}^{1} f^{q-1}\left(\tau, \tau^{\alpha-1}, \tau^{\alpha-\gamma-1}\right) d \tau<+\infty
$$

then the p-Laplacian fractional order differential equation (1.1) has a unique positive solution $z^{*}(t)$. Let $y^{*}(t)=\mathcal{D}_{\boldsymbol{t}}^{\gamma} z^{*}(t)$, then $y^{*} \in D$. Moreover for any initial value $y_{0} \in D$, the sequence of functions defined by

$$
y_{n}=\int_{0}^{1} H(t, s) \varphi_{q}\left(\int_{0}^{1} G_{\beta}(s, \tau) f\left(\tau, I^{\gamma} y_{n-1}(\tau), y_{n-1}(\tau)\right) d \tau\right) d s, n \geq 1
$$

converges uniformly to $y^{*}(t)=\mathcal{D}_{\boldsymbol{t}}^{\gamma} z^{*}(t)$ on $[0,1]$ as $n \rightarrow+\infty$. Furthermore there exists an error estimation

$$
\left\|z_{n}-z^{*}\right\| \leq \frac{2}{\Gamma(\gamma+1) \sqrt{\epsilon}}\left(1-\epsilon^{(q-1)^{n}}\right)\left\|y_{0}\right\|,
$$

which has the rate of convergence

$$
\left\|z_{n}-z^{*}\right\|=o\left(1-\epsilon^{(q-1)^{n}}\right)
$$

where $0<\epsilon<1$ is a positive constant which is determined by the initial value $y_{0}$.

Proof. It follows from Lemma 2 that we can define a nonlinear operator $T$ : $D \rightarrow E$ by

$$
(T x)(t)=\int_{0}^{1} H(t, s) \varphi_{q}\left(\int_{0}^{1} G_{\beta}(s, \tau) f\left(\tau, I^{\gamma} x(\tau), x(\tau)\right) d \tau\right) d s
$$


and a fixed point $x$ of the operator $T$ is a solution of the fractional order integrodifferential equation (2.4). Thus $z(t)=I^{\gamma} x(t)$ is a solution of the $p$-Laplacian fractional order differential equation (1.1).

In what follows, we prove that $T$ is well defined and $T: D \rightarrow D$. In fact, according to the definition of $D$, for any $x \in D$, there are two numbers $K_{x}>$ $1>k_{x}>0$ such that

$$
k_{x} t^{\alpha-\gamma-1} \leq x(t) \leq K_{x} t^{\alpha-\gamma-1}, t \in[0,1] .
$$

Consequently, one has

$$
\begin{aligned}
& \frac{k_{x} \Gamma(\alpha-\gamma)}{\Gamma(\alpha)} t^{\alpha-1}=\frac{K_{x}}{\Gamma(\gamma)} \int_{0}^{t}(t-s)^{\gamma-1} s^{\alpha-\gamma-1} d s \leq I^{\gamma} x(t)=\frac{1}{\Gamma(\gamma)} \\
& \times \int_{0}^{t}(t-s)^{\gamma-1} x(s) d s \leq \frac{K_{x}}{\Gamma(\gamma)} \int_{0}^{t}(t-s)^{\gamma-1} s^{\alpha-\gamma-1} d s=\frac{K_{x} \Gamma(\alpha-\gamma)}{\Gamma(\alpha)} t^{\alpha-1}
\end{aligned}
$$

Thus applying Lemma 3, (3.1) and (3.2)-(3.4), we have

$$
\begin{aligned}
& \int_{0}^{1} H(t, s) \varphi_{q}\left(\int_{0}^{1} G_{\beta}(s, \tau) f\left(\tau, I^{\gamma} x(\tau), x(\tau)\right) d \tau\right) d s \\
& \leq e\left(\frac{\beta-1}{\Gamma(\beta)}\right)^{q-1} \int_{0}^{1}\left[f\left(\tau, \frac{K_{x} \Gamma(\alpha-\gamma)}{\Gamma(\alpha)} \tau^{\alpha-1}, K_{x} \tau^{\alpha-\gamma-1}\right)\right]^{q-1} d \tau t^{\alpha-\gamma-1} \\
& \leq e\left(\frac{\beta-1}{\Gamma(\beta)}\right)^{q-1} \sigma^{q-1}\left(K_{x} \max \left(\frac{\Gamma(\alpha-\gamma)}{\Gamma(\alpha)}, 1\right)\right) \\
& \quad \times \int_{0}^{1} f^{q-1}\left(\tau, \tau^{\alpha-1}, \tau^{\alpha-\gamma-1}\right) d \tau t^{\alpha-\gamma-1}<\infty
\end{aligned}
$$

On the other hand, we also have

$$
\begin{aligned}
& \int_{0}^{1} H(t, s) \varphi_{q}\left(\int_{0}^{1} G_{\beta}(s, \tau) f\left(\tau, I^{\gamma} x(\tau), x(\tau)\right) d \tau\right) d s \\
& \geq \frac{d}{\Gamma(\beta)} \int_{0}^{1} \mathcal{G}_{A}(s) s^{(\beta-1)(q-1)}(1-s)^{q-1} d s \int_{0}^{1} \tau^{q-1}(1-\tau)^{(\beta-1)(q-1)} \\
& \quad \times f^{q-1}\left(\tau, \frac{k_{x} \Gamma(\alpha-\gamma)}{\Gamma(\alpha)} \tau^{\alpha-1}, k_{x} \tau^{\alpha-\gamma-1}\right) d \tau t^{\alpha-\gamma-1} \\
& \geq \\
& \quad \frac{d}{\Gamma(\beta)} \sigma^{q-1}\left(k_{x} \min \left(\frac{\Gamma(\alpha-\gamma)}{\Gamma(\alpha)}, 1\right)\right) \int_{0}^{1} \mathcal{G}_{A}(s) s^{(\beta-1)(q-1)}(1-s)^{q-1} d s \\
& \quad \times \int_{0}^{1} \tau^{q-1}(1-\tau)^{(\beta-1)(q-1)} f^{q-1}\left(\tau, \tau^{\alpha-1}, \tau^{\alpha-\gamma-1}\right) d \tau t^{\alpha-\gamma-1} .
\end{aligned}
$$

(3.5)-(3.6) yield that $T$ is well defined and $T(D) \subset D$.

Now given $y_{0} \in D$, there exist four positive constants $k_{y_{0}}, K_{y_{0}}, \widetilde{k}_{y_{0}}, \widetilde{K}_{y_{0}}$ such that

$$
k_{y_{0}} t^{\alpha-\gamma-1} \leq y_{0} \leq K_{y_{0}} t^{\alpha-\gamma-1}, \quad \widetilde{k}_{y_{0}} t^{\alpha-\gamma-1} \leq T y_{0} \leq \widetilde{K}_{y_{0}} t^{\alpha-\gamma-1}
$$


which implies that

$$
\frac{\widetilde{k}_{y_{0}}}{K_{y_{0}}} y_{0} \leq T y_{0} \leq \frac{\widetilde{K}_{y_{0}}}{k_{y_{0}}} y_{0} .
$$

Choose a constant $t_{0}$ such that

$$
0<t_{0} \leq \min \left\{\left(\widetilde{k}_{y_{0}} / K_{y_{0}}\right)^{\frac{1}{2-q}},\left(k_{y_{0}} / \widetilde{K}_{y_{0}}\right)^{\frac{1}{2-q}}\right\}
$$

and then we have $t_{0} \in(0,1)$ and

$$
t_{0}^{2-q} y_{0} \leq T y_{0} \leq\left(\frac{1}{t_{0}}\right)^{2-q} y_{0} .
$$

Take $u_{0}=t_{0} y_{0}, v_{0}=y_{0} / t_{0}, t_{0} \in(0,1)$. Clearly $u_{0} \leq v_{0}$. Now we define the iterative sequence as follows

$$
u_{n}=T u_{n-1}, v_{n}=T v_{n-1},(n=1,2, \ldots) .
$$

By (A), we know that $T$ is an increasing operator in $x$ and

$$
\begin{aligned}
& T(r x)=\int_{0}^{1} H(t, s) \varphi_{q}\left(\int_{0}^{1} G_{\beta}(s, \tau) f\left(\tau, r I^{\gamma} x(\tau), r x(\tau)\right) d \tau\right) d s \\
& \geq \sigma^{q-1}(r) \int_{0}^{1} H(t, s) \varphi_{q}\left(\int_{0}^{1} G_{\beta}(s, \tau) f\left(\tau, I^{\gamma} x(\tau), x(\tau)\right) d \tau\right) d s \\
& =\sigma^{q-1}(r) T x, \quad 0<r<1 \\
& T(r x)=\int_{0}^{1} H(t, s) \varphi_{q}\left(\int_{0}^{1} G_{\beta}(s, \tau) f\left(\tau, r I^{\gamma} x(\tau), r x(\tau)\right) d \tau\right) d s \\
& \leq \sigma^{1-q}\left(\frac{1}{r}\right) \int_{0}^{1} H(t, s) \varphi_{q}\left(\int_{0}^{1} G_{\beta}(s, \tau) f\left(\tau, I^{\gamma} x(\tau), x(\tau)\right) d \tau\right) d s \\
& =\sigma^{1-q}\left(\frac{1}{r}\right) T x, \quad r \geq 1
\end{aligned}
$$

It follows from (3.7)-(3.9) that

$$
\begin{aligned}
& u_{1}=T u_{0} \geq \sigma^{q-1}\left(t_{0}\right) T y_{0} \geq t_{0}^{q-1} T y_{0} \geq t_{0} y_{0}=u_{0}, \\
& v_{1}=T v_{0} \leq\left(\frac{1}{\sigma\left(t_{0}\right)}\right)^{q-1} T y_{0} \leq\left(\frac{1}{t_{0}}\right)^{q-1} T y_{0} \leq \frac{1}{t_{0}} y_{0}=v_{0} .
\end{aligned}
$$

Thus from induction, (3.10) and $u_{0} \leq v_{0}$, one has

$$
u_{0} \leq u_{1} \leq \cdots \leq u_{n} \leq \cdots \leq v_{n} \leq \cdots \leq v_{1} \leq v_{0} .
$$

Since $u_{0}=t_{0}^{2} v_{0}$, we get

$$
u_{1}=T u_{0}=T\left(t_{0}^{2} v_{0}\right) \geq \sigma^{q-1}\left(t_{0}^{2}\right) T v_{0} \geq\left(t_{0}^{2}\right)^{q-1} v_{1} .
$$


Thus by induction, we have $u_{n} \geq\left(t_{0}^{2}\right)^{(q-1)^{n}} v_{n},(n=0,1,2, \ldots)$. Since $P$ is a normal cone with normality constant 1 , and $u_{n+m}-u_{n} \leq v_{n}-u_{n}$, for any $m \in N$, we have

$$
\left\|u_{n+m}-u_{n}\right\| \leq\left\|v_{n}-u_{n}\right\| \leq\left(1-\left(t_{0}^{2}\right)^{(q-1)^{n}}\right)\left\|v_{0}\right\| \rightarrow 0, \text { as } n \rightarrow+\infty,
$$

which implies that $\left\{u_{n}\right\}$ is a Cauchy sequence, and $u_{n}$ converges to some $y^{*} \in$ $D$. By (3.12) and

$$
\left\|v_{n}-y^{*}\right\| \leq\left\|v_{n}-u_{n}\right\|+\left\|u_{n}-y^{*}\right\|
$$

we also obtain $v_{n} \rightarrow y^{*}$. It follows from (3.11) that $y^{*} \in D$ is a fixed point of $T$, and $y^{*} \in\left[u_{0}, v_{0}\right]$.

Thus for any initial value $y_{0} \in D$, it follows from $u_{0} \leq y_{0} \leq v_{0}$ that $u_{n} \leq y_{n} \leq v_{n},(n=1,2, \ldots)$. So we have

$$
\begin{aligned}
\left\|y_{n}-y^{*}\right\| & \leq\left\|y_{n}-u_{n}\right\|+\left\|u_{n}-y^{*}\right\| \leq 2\left\|v_{n}-u_{n}\right\| \\
& \leq 2\left(1-\left(t_{0}^{2}\right)^{(q-1)^{n}}\right)\left\|v_{0}\right\|,
\end{aligned}
$$

which implies that the sequence of functions defined by

$$
y_{n}=\int_{0}^{1} H(t, s) \varphi_{q}\left(\int_{0}^{1} G_{\beta}(s, \tau) f\left(\tau, I^{\gamma} y_{n-1}(\tau), y_{n-1}(\tau)\right) d \tau\right) d s, n=1,2, \ldots
$$

converges uniformly to the positive solution $y^{*}(t)$ of equation $(2.4)$ on $[0,1]$ as $n \rightarrow+\infty$. Furthermore we have the error estimation

$$
\left\|y_{n}-y^{*}\right\| \leq \frac{2}{\sqrt{\epsilon}}\left(1-\epsilon^{(q-1)^{n}}\right)\left\|y_{0}\right\|,
$$

which has the rate of convergence

$$
\left\|y_{n}-y^{*}\right\|=o\left(1-\epsilon^{(q-1)^{n}}\right)
$$

where $0<\epsilon=t_{0}^{2}<1$ is a constant which is determined by $y_{0}$.

Next we prove that the positive solution of the problem (2.4) is unique. Actually, suppose $\bar{y} \in D$ is any fixed point of $T$, then we have $T \bar{y}=\bar{y}$. From $\bar{y}, y^{*} \in D$ and the definition of $D$, let $t_{1}=\sup \left\{t>0: \bar{y} \geq t y^{*}\right\}$, then we have $0<t_{1}<+\infty$. We assert that $t_{1} \geq 1$. If not, we have $0<t_{1}<1$, thus

$$
\bar{y}=T \bar{y} \geq T\left(t_{1} y^{*}\right) \geq \sigma^{q-1}\left(t_{1}\right) T y^{*}=\sigma^{q-1}\left(t_{1}\right) y^{*} \geq t_{1}^{q-1} y^{*},
$$

since $1<q<2$ and we have $t_{1}^{q-1}>t_{1}$, a contradiction. Thus we obtain that $t_{1} \geq 1$ and $\bar{y} \geq y^{*}$. In the same way, we also have $\bar{y} \leq y^{*}$, that is $\bar{y}=y^{*}$, and then $y^{*}$ is a unique fixed point of $T$ in $D$. Of course, it is also a unique positive solution of the fractional order $p$-Laplacian differential equation (2.4).

In the end, let $z^{*}(t)=I^{\gamma} y^{*}(t)$, then $z^{*}$ is unique positive solution of the $p$-Laplacian fractional order differential equation (1.1). Moreover for any initial value $y_{0} \in D$, the sequence of functions defined by

$$
y_{n}=\int_{0}^{1} H(t, s) \varphi_{q}\left(\int_{0}^{1} G_{\beta}(s, \tau) f\left(\tau, I^{\gamma} y_{n-1}(\tau), y_{n-1}(\tau)\right) d \tau\right) d s, n=1,2, \ldots
$$


converges uniformly to $y^{*}(t)=\mathcal{D}_{\boldsymbol{t}}^{\gamma} z^{*}(t)$ on $[0,1]$ as $n \rightarrow+\infty$. Furthermore there exists an error estimation

$$
\left\|z_{n}-z^{*}\right\| \leq \frac{2}{\Gamma(\gamma+1) \sqrt{\epsilon}}\left(1-\epsilon^{(q-1)^{n}}\right)\left\|y_{0}\right\|,
$$

which has the rate of convergence $\left\|z_{n}-z^{*}\right\|=o\left(1-\epsilon^{(q-1)^{n}}\right)$, where $0<\epsilon<1$ is a positive constant which is determined by the initial value $y_{0}$.

Remark 4. In Theorem 1, we derive a new result on the sufficient condition for the existence and uniqueness of a positive solution for a fractional order $p$ Laplacian differential equation with nonlocal boundary value condition. More important, we construct an iterative sequence for the solution and give estimation of the error and the rate of convergence. In particular, the initial value of the iterative sequence can be chosen arbitrarily in $D$, thus we can choose some simple initial value such 0 or $t^{\alpha-\gamma-1}$, which is very beneficial to our computation.

Remark 5. Theorem 1 is still valid if $(\mathbf{A})$ is replaced by one of the following assumptions:

$\left(\mathbf{H}_{1}^{*}\right) f:((0,1) \times[0,+\infty) \times[0,+\infty) \rightarrow[0,+\infty))$ is continuous and nondecreasing in the second and the third variables; and there exists a constant $0<b<1$ such that, for any $(t, x, y) \in(0,1) \times[0,+\infty) \times[0,+\infty)$ and $r \in(0,1)$,

$$
f(t, r x, r y) \geq r^{b} f(t, x, y) .
$$

$\left(\mathbf{H}_{2}^{*}\right) f:((0,1) \times[0,+\infty) \times[0,+\infty) \rightarrow[0,+\infty))$ is continuous and nondecreasing in the second and the third variables; and for any $r \in(0,1)$, there exists $\eta(r)=m\left(r^{-\kappa}-1\right)$ such that, for all $(t, x, y) \in(0,1) \times[0,+\infty) \times[0,+\infty)$, $0<m \leq 1,0<\kappa<1$,

$$
f(t, r x, r y) \geq r[1+\eta(r)] f(t, x, y) .
$$

Remark 6 . The short memory behaviors often happen in various biomedicine processes [3] and porous medium flow with short wave characters [1], here we also comment the $p$-Laplacian fractional order differential equation (1.1) has unique positive solution with similar iterative properties and estimation of error as Theorem 1 when $t$ is in a short memory measure such as $t \in(1-\epsilon, 1)$ with $\lim _{\epsilon \rightarrow 1} \epsilon=1$ since the Green function possesses the same characters.

\section{Numerical results}

Example 1. Consider the existence of positive solutions for the nonlinear fractional differential equation

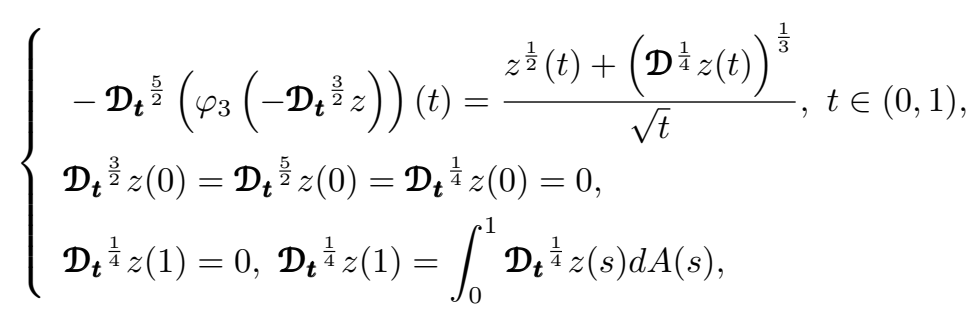


where $A$ is a function of bounded variation

$$
A(t)=\left\{\begin{array}{l}
0, \quad t \in[0,1 / 2), \\
3 / 2, \quad t \in[1 / 2,3 / 4), \\
1, \quad t \in[3 / 4,1]
\end{array}\right.
$$

Then by simple computation, the equation (4.1) is equivalent to a 4-point boundary value problem with coefficients of both signs

$$
\left\{\begin{array}{l}
-\mathcal{D}^{\frac{5}{2}}\left(\varphi_{3}\left(-\mathcal{D}_{t}^{\frac{3}{2}} z\right)\right)(t)=\frac{z^{\frac{1}{2}}(t)+\left(\mathcal{D}^{\frac{1}{4}} z(t)\right)^{\frac{1}{3}}}{\sqrt{t}}, t \in(0,1), \\
\mathcal{D}^{\frac{3}{2}} z(0)=\mathcal{D}_{\boldsymbol{t}^{\frac{5}{2}}} z(0)=\mathcal{D}_{\boldsymbol{t}}^{\frac{1}{4}} z(0)=0, \mathcal{D}_{\boldsymbol{t}}^{\frac{1}{4}} z(1)=0 \\
\mathcal{D}_{\boldsymbol{t}}^{\frac{1}{4}} z(1)=1.5 \mathcal{D}_{\boldsymbol{t}^{\frac{1}{4}}} z(0.5)-0.5 \mathcal{D}_{\boldsymbol{t}}^{\frac{1}{4}} z(0.75)
\end{array}\right.
$$

Thus we only need to study the 4-point boundary value problem (4.2).

Let $f(t, x, y)=\left(x^{\frac{1}{2}}+y^{\frac{1}{3}}\right) / t$, then $f \in C((0,1) \times[0,+\infty) \times[0, \infty),[0,+\infty))$, and for any fixed $t \in(0,1), f(t, x, y)$ is nondecreasing in $x$ and $y$. Now take $\sigma(r)=r^{\frac{3}{4}}, r \in[0,1]$, then we have $\sigma(r)>r$ for $r \in(0,1)$, and for any $r \in(0,1)$ and $(0,1) \times[0,+\infty) \times[0, \infty)$, we have

$$
f(t, r x, r y)=\frac{r^{\frac{1}{2}} x^{\frac{1}{2}}+r^{\frac{1}{3}} y^{\frac{1}{3}}}{\sqrt{t}} \geq r^{\frac{3}{4}} \frac{x^{\frac{1}{2}}+y^{\frac{1}{3}}}{\sqrt{t}}=r^{\frac{3}{4}} f(t, x, y) .
$$

Thus the condition (A) is satisfied.

Next we verify the condition (B). Let $\alpha=\frac{3}{2}, \beta=\frac{5}{2}, \gamma=\frac{1}{4}, p=3$, then $0<\gamma \leq 1<\alpha \leq 2<\beta<3, \alpha-\gamma>1$ and

$$
G_{\alpha-\gamma}(t, s)=\frac{1}{\Gamma(5 / 4)}\left\{\begin{array}{l}
{[t(1-s)]^{\frac{1}{4}}-(t-s)^{\frac{1}{4}}=: G_{1}(t, s), 0 \leq s \leq t \leq 1} \\
{[t(1-s)]^{\frac{1}{4}}=: G_{2}(t, s), \quad 0 \leq t \leq s \leq 1}
\end{array}\right.
$$

Thus, we have

$$
\begin{aligned}
& \mathcal{G}_{A}(s)=\int_{0}^{1} G_{\alpha-\gamma}(t, s) d A(t) \\
& =\frac{1}{\Gamma\left(\frac{5}{4}\right)} \begin{cases}1.5 G_{1}(0.5, s)-0.5 G_{1}(0.75, s), & 0 \leq s<0.5, \\
1.5 G_{2}(0.5, s)-0.5 G_{1}(0.75, s), & 0.5 \leq s<0.75, \\
1.5 G_{2}(0.5, s)-0.5 G_{2}(0.75, s), & 0.75 \leq s \leq 1,\end{cases} \\
& =\frac{1}{\Gamma\left(\frac{5}{4}\right)} \begin{cases}\frac{3-\sqrt[4]{3}}{2^{5}}(1-s)^{\frac{1}{4}}+\frac{1}{2}\left(\frac{3}{4}-s\right)^{\frac{1}{4}}-\frac{3}{2}\left(\frac{1}{2}-s\right)^{\frac{1}{4}}, & 0 \leq s<\frac{1}{2}, \\
\frac{3-\sqrt[4]{3}}{2^{5}}(1-s)^{\frac{1}{4}}+\frac{1}{2}\left(\frac{3}{4}-s\right)^{\frac{1}{4}}, & \frac{1}{2} \leq s<\frac{3}{4}, \\
\frac{3-\sqrt[4]{3}}{2^{5}}(1-s)^{\frac{1}{4}}, & \frac{3}{4} \leq s \leq 1,\end{cases}
\end{aligned}
$$

which implies that $\mathcal{G}_{A}(s) \geq 0$ for $s \in[0,1]$. 
On the other hand, we have

$$
\mathcal{A}=\int_{0}^{1} t^{\alpha-\gamma-1} d A(t)=\int_{0}^{1} t^{\frac{1}{4}} d A(t)=1-\frac{1}{4} \int_{0}^{1} A(t) t^{-\frac{3}{4}} d t=0.7961<1 .
$$

Therefore, the condition (B) is also satisfied.

Now we check the condition (3.1). In fact,

$$
\begin{aligned}
0 & <\int_{0}^{1} f^{q-1}\left(\tau, \tau^{\alpha-1}, \tau^{\alpha-\gamma-1}\right) d \tau=\int_{0}^{1}\left(\frac{\tau^{\frac{1}{4}}+\tau^{\frac{1}{12}}}{\sqrt{\tau}}\right)^{\frac{3}{2}} d \tau \\
& <2^{\frac{3}{2}} \int_{0}^{1} \tau^{-\frac{15}{24}} d \tau<+\infty .
\end{aligned}
$$

Then the $p$-Laplacian fractional order differential equation (1.1) has a unique positive solution $z^{*}(t)=I^{\frac{1}{4}} y^{*}(t), y^{*} \in D$. Moreover for any initial value $y_{0} \in D$, the sequence of functions defined by

$$
\begin{aligned}
y_{n} & =\int_{0}^{1}\left(\frac{\mathcal{G}_{A}(s)}{0.2039} t^{\frac{1}{4}}+G_{\frac{5}{4}}(t, s)\right) \\
& \times\left(\int_{0}^{1} G_{\frac{5}{2}}(s, \tau) \frac{\left(I^{\frac{1}{4}} y_{n-1}(\tau)\right)^{\frac{1}{2}}+\left(y_{n-1}(\tau)\right)^{\frac{1}{3}}}{\sqrt{\tau}} d \tau\right)^{\frac{3}{2}} d s, \quad n=1,2, \ldots
\end{aligned}
$$

converges uniformly to $y^{*}(t)$ on $[0,1]$ as $n \rightarrow+\infty$. Furthermore there exists an error estimation

$$
\left\|z_{n}-z^{*}\right\|=\max _{t \in[0,1]}\left|I^{\frac{1}{4}}\left(y_{n}(t)-y^{*}(t)\right)\right| \leq \frac{4}{\Gamma\left(\frac{1}{4}\right)}\left\|y_{n}-y^{*}\right\| \leq \frac{8}{\Gamma\left(\frac{1}{4}\right) \sqrt{\epsilon}}\left(1-\epsilon^{\left(\frac{1}{2}\right)^{n}}\right)\left\|y_{0}\right\|,
$$

which has the rate of convergence

$$
\left\|z_{n}-z^{*}\right\|=o\left(1-\epsilon^{\left(\frac{1}{2}\right)^{n}}\right),
$$

where $0<\epsilon<1$ is a positive constant which is determined by the initial value $y_{0}$. In particular, if $y_{0}=t^{\frac{1}{4}}$, then by computation, we have

$$
0.2513 t^{\frac{1}{4}} \leq T y_{0} \leq 5.6577 t^{\frac{1}{4}} \text {. }
$$

Take

$$
t_{0}=\min \left\{(0.2513)^{2},\left(\frac{1}{5.6577}\right)^{2}\right\}=\min \{0.0632,0.0313\}=0.0313,
$$

then we have the error estimation

$$
\left\|z_{n}-z^{*}\right\| \leq 70.4846\left(1-0.00097969^{\left(\frac{1}{2}\right)^{n}}\right),
$$

and the estimate of convergence rate by

$$
\left\|z_{n}-z^{*}\right\|=o\left(1-0.00097969^{\left(\frac{1}{3}\right)^{n}}\right) .
$$

Table 1 shows the maximum errors of the iterative scheme compared to the exact solution. The numerical results confirm that the scheme (4.3) has the global convergence rate $o\left(1-0.00097969^{\left(\frac{1}{2}\right)^{n}}\right)$. 
Table 1. Numerical results of maximum errors while $n$ increases.

\begin{tabular}{lc}
\hline $\mathrm{n}$ & Maximum errors for $\alpha=\frac{3}{2}, \beta=\frac{5}{2}, \gamma=\frac{1}{4}, p=3$ \\
\hline 15 & 0.0149 \\
20 & $4.6571 \mathrm{e}-004$ \\
25 & $1.4554 \mathrm{e}-005$ \\
30 & $4.5480 \mathrm{e}-007$ \\
\hline
\end{tabular}

\section{Conclusions}

By introducing a more general growth condition, a new result on the uniqueness of positive solutions for the general $p$-Laplacian fractional order differential equation is established. Through a detailed theoretical analysis, we construct an iterative converging scheme and also obtain maximum error estimates and the convergence rate. The developed algorithms, theoretical and numerical results can also be extended to the problems discussed in $[10,19,21]$.

\section{Acknowledgements}

The authors were supported financially by the National Natural Science Foundation of China (11571296), and the Australia Research Council.

\section{References}

[1] L. Caffarelli and J.L. Vazquez. Nonlinear porous medium flow with fractional potential pressure. Archive for Rational Mechanics and Analysis, 202(2):537565, 2011. https://doi.org/10.1007/s00205-011-0420-4.

[2] Y. Cui and Y. Zou. Monotone iterative method for differential systems with coupled integral boundary value problems. Boundary Value Problems, 2013:245, 2013. https://doi.org/10.1186/1687-2770-2013-245.

[3] H.A.A. El-Saka. The fractional-order sis epidemic model with variable population size. Journal of the Egyptian Mathematical Society, 22(1):50-54, 2014. ISSN 1110-256X. https://doi.org/10.1016/j.joems.2013.06.006.

[4] L. Guo, L. Liu and Y. Wu. Existence of positive solutions for singular fractional differential equations with infinite-point boundary conditions. Nonlinear Analysis: Modelling and Control, 21(5):635-650, 2016. https://doi.org/10.15388/NA.2016.5.5.

[5] X. Hao, H. Wang, L. Liu and Y. Cui. Positive solutions for a system of nonlinear fractional nonlocal boundary value problems with parameters and p-Laplacian operator. Boundary Value Problems, 2017(1):182, 2017. https://doi.org/10.1186/s13661-017-0915-5.

[6] J. Jiang, L. Liu and Y. Wu. Positive solutions to singular fractional differential system with coupled boundary conditions. Communications in Nonlinear Science and Numerical Simulation, 18(11):3061-3074, 2013. https://doi.org/10.1016/j.cnsns.2013.04.009. 
[7] J. Liu and A. Qian. Ground state solution for a Schrödinger-Poisson equation with critical growth. Nonlinear Analysis: Real World Applications, 40:428-443, 2018. https://doi.org/10.1016/j.nonrwa.2017.09.008.

[8] A. Mao and H. Chang. Kirchhoff type problems in $r^{n}$ with radial potentials and locally lipschitz functional. Applied Mathematics Letters, 62:49-54, 2016. https://doi.org/10.1016/j.aml.2016.06.014.

[9] A. Mao and W. Wang. Nontrivial solutions of nonlocal fourth order elliptic equation of Kirchhoff type in $\mathrm{R}^{3}$. Journal of Mathematical Analysis and Applications, 459(1):556-563, 2018. https://doi.org/10.1016/j.jmaa.2017.10.020.

[10] J. Mao, Z. Zhao and N. Xu. On existence and uniqueness of positive solutions for integral boundary boundary value problems. Electronic Journal of Qualitative Theory of Differential Equations, 16:1-8, 2010. https://doi.org/10.14232/ejqtde.2010.1.16.

[11] K. Miller and B. Ross. An Introduction to the Fractional Calculus and Fractional Differential Equations. Wiley, New York, 1993.

[12] T. Ren, S. Li, X. Zhang and L. Liu. Maximum and minimum solutions for a nonlocal $p$-Laplacian fractional differential system from eco-economical processes. Boundary Value Problems, 2017(1):118, 2017. https://doi.org/10.1186/s13661017-0849-y.

[13] M. Shao and A. Mao. Multiplicity of solutions to Schrödinger-Poisson system with concave-convex nonlinearities. Applied Mathematics Letters, 83:212-218, 2018. https://doi.org/10.1016/j.aml.2018.04.005.

[14] Y. Wang, L. Liu and Y. Wu. Positive solutions for a class of fractional boundary value problem with changing sign nonlinearity. Nonlinear Analysis: Theory, Methods \& Applications, 74(17):6434-6441, 2011. https://doi.org/10.1016/j.na.2011.06.026.

[15] Y. Wang, L. Liu, X. Zhang and Y. Wu. Positive solutions of an abstract fractional semipositone differential system model for bioprocesses of HIV infection. Applied Mathematics and Computation, 258:312-324, 2015. https://doi.org/10.1016/j.amc.2015.01.080.

[16] J.R.L. Webb and M. Zima. Multiple positive solutions of resonant and non-resonant nonlocal boundary value problems. Nonlinear Analysis: Theory, Methods \& Applications, 71(3-4):1369-1378, 2009. https://doi.org/10.1016/j.na.2008.12.010.

[17] J. Wu, X. Zhang, L. Liu and Y. Wu. Positive solution of singular fractional differential system with nonlocal boundary conditions. Advances in Difference Equations, 2014(1):323, 2014. https://doi.org/10.1186/1687-1847-2014-323.

[18] J. Wu, X. Zhang, L. Liu and Y. Wu. Twin iterative solutions for a fractional differential turbulent flow model. Boundary Value Problems, 2016(1):98, 2016. https://doi.org/10.1186/s13661-016-0604-9.

[19] J. Wu, X. Zhang, L. Liu, Y. Wu and Y. Cui. The convergence analysis and error estimation for unique solution of a p-Laplacian fractional differential equation with singular decreasing nonlinearity. Boundary Value Problems, 2018(82), 2018. https://doi.org/10.1186/s13661-018-1003-1.

[20] J. Wu, X. Zhang, L. Liu, Y. Wu and B. Wiwatanapataphee. Iterative algorithm and estimation of solution for a fractional order differential equation. Boundary Value Problems, 2016(1):116, 2016. https://doi.org/10.1186/s13661-016-06085 . 
[21] X. Zhang and L. Liu. A necessary and sufficient condition of positive solutions for nonlinear singular differential systems with four-point boundary conditions. Applied Mathematics and Computation, 215(10):3501-3508, 2010. https://doi.org/10.1016/j.amc.2009.10.044.

[22] X. Zhang, L. Liu, B. Wiwatanapataphee and Y. Wu. The eigenvalue for a class of singular $p$-Laplacian fractional differential equations involving the RiemannStieltjes integral boundary condition. Applied Mathematics and Computation, 235:412-422, 2014. https://doi.org/10.1016/j.amc.2014.02.062.

[23] X. Zhang, L. Liu and Y. Wu. The eigenvalue problem for a singular higher fractional differential equation involving fractional derivatives. Applied Mathematics and Computation, 218:8526-8536, 2012. https://doi.org/10.1016/j.amc.2012.02.014.

[24] X. Zhang, L. Liu and Y. Wu. Existence results for multiple positive solutions of nonlinear higher order perturbed fractional differential equations with derivatives. Applied Mathematics and Computation, 219:1420-1433, 2012. https://doi.org/10.1016/j.amc.2012.07.046.

[25] X. Zhang, L. Liu and Y. Wu. Multiple positive solutions of a singular fractional differential equation with negatively perturbed term. Mathematical and Computer Modelling, 55(3-4):1263-1274, 2012. https://doi.org/10.1016/j.mcm.2011.10.006.

[26] X. Zhang, L. Liu and Y. Wu. The uniqueness of positive solution for a fractional order model of turbulent flow in a porous medium. Applied Mathematics Letters, 37:26-33, 2014. https://doi.org/10.1016/j.aml.2014.05.002.

[27] X. Zhang, L. Liu and Y. Wu. Variational structure and multiple solutions for a fractional advection-dispersion equation. Computers \& Mathematics with Applications, 68(12, Part A):1794-1805, 2014. https://doi.org/10.1016/j.camwa.2014.10.011.

[28] X. Zhang, L. Liu and Y. Wu. The entire large solutions for a quasilinear Schrödinger elliptic equation by the dual approach. Applied Mathematics Letters, 55:1-9, 2016. https://doi.org/10.1016/j.aml.2015.11.005.

[29] X. Zhang, L. Liu, Y. Wu and L. Caccetta. Entire large solutions for a class of Schrödinger systems with a nonlinear random operator. Journal of Mathematical Analysis and Applications, 423(2):1650-1659, 2015. https://doi.org/10.1016/j.jmaa.2014.10.068.

[30] X. Zhang, L. Liu, Y. Wu and Y. Cui. Entire blow-up solutions for a quasilinear pLaplacian Schrödinger equation with a non-square diffusion term. Applied Mathematics Letters, 74:85-93, 2017. https://doi.org/10.1016/j.aml.2017.05.010.

[31] X. Zhang, L. Liu, Y. Wu and Y. Cui. New result on the critical exponent for solution of an ordinary fractional differential problem. Journal of Function Spaces, 1:3976469, 2017. https://doi.org/10.1155/2017/3976469.

[32] X. Zhang, L. Liu, Y. Wu and Y. Cui. The existence and nonexistence of entire large solutions for a quasilinear Schrödinger elliptic system by dual approach. Journal of Mathematical Analysis and Applications, 464(2):1089-1106, 2018. https://doi.org/10.1016/j.jmaa.2018.04.040.

[33] X. Zhang, L. Liu, Y. Wu and Y. Cui. Existence of infinitely solutions for a modified nonlinear schrodinger equation via dual approach. Electronic Journal of Differential Equations, 2018(147):1-15, 2018. 
[34] X. Zhang, L. Liu, Y. Wu and Y. Lu. The iterative solutions of nonlinear fractional differential equations. Applied Mathematics and Computation, 219(9):46804691, 2013. https://doi.org/10.1016/j.amc.2012.10.082.

[35] X. Zhang, L. Liu, Y. Wu and B. Wiwatanapataphee. The spectral analysis for a singular fractional differential equation with a signed measure. Applied Mathematics and Computation, 257:252-263, 2015. https://doi.org/10.1016/j.amc.2014.12.068.

[36] X. Zhang, L. Liu, Y. Wu and B. Wiwatanapataphee. Nontrivial solutions for a fractional advection dispersion equation in anomalous diffusion. Applied Mathematics Letters, 66:1-8, 2017. https://doi.org/10.1016/j.aml.2016.10.015.

[37] X. Zhang, C. Mao, L. Liu and Y. Wu. Exact iterative solution for an abstract fractional dynamic system model for bioprocess. Qualitative Theory of Dynamical Systems, 16(1):205-222, 2017. https://doi.org/10.1007/s12346-015-0162-z.

[38] X. Zhang, Y. Wu and Y. Cui. Existence and nonexistence of blow-up solutions for a Schrödinger equation involving a nonlinear operator. Applied Mathematics Letters, 82:85-91, 2018. https://doi.org/10.1016/j.aml.2018.02.019.

[39] Z. Zhao. Existence and uniqueness of fixed points for some mixed monotone operators. Nonlinear Analysis: Theory, Methods \& Applications, 73(6):14811490, 2010. https://doi.org/10.1016/j.na.2010.04.008.

[40] B. Zhu, L. Liu and Y. Wu. Local and global existence of mild solutions for a class of nonlinear fractional reaction-diffusion equations with delay. Applied Mathematics Letters, 61:73-79, 2016. https://doi.org/10.1016/j.aml.2016.05.010.

[41] B. Zhu, L. Liu and Y. Wu. Local and global existence of mild solutions for a class of semilinear fractional integro-differential equations. Fractional Calculus and Applied Analysis, 20(6):1338-1355, 2017. https://doi.org/10.1515/fca-20170071.

[42] M. Zuo, X. Hao, L. Liu and Y. Cui. Existence results for impulsive fractional integro-differential equation of mixed type with constant coefficient and antiperiodic boundary conditions. Boundary Value Problems, 2017(1):161, 2017. https://doi.org/10.1186/s13661-017-0892-8. 